\title{
A QUADRANGULAR PRISM OF SELF INVISIBILITY
}

\author{
Yizong $\mathrm{He}$ \\ yizonghe@126.com \\ Department of Electrical and Computer Engineering, \\ University of Minnesota, \\ Minneapolis, MN 55455, USA
}

\begin{abstract}
A quadrangular prism of self-invisibility is originally reported in this paper. It is a self-invisible unit, which does not depend on any outer refractive materials or reflective mirrors. Four identical triangular prisms with the lateral faces of mirrors, divided into two groups, are symmetrically placed on two transparent rectangular prisms in a symmetrical way. Two identical rectangular prisms serve as the lateral faces of a quadrangular prism. Two incident beams, parallel to the rectangular prisms, will be reflected by relevant eight mirror faces of all the triangular prisms, in sequence out of the whole system as if the prisms do not exist as long as all the base angles of triangular prisms are adjusted accurately. One of the potential applications will be mentioned briefly.
\end{abstract}

\section{KEYWORDS}

Stealth Technology, Camouflage Technology, Invisible, Automobile Industry

\section{INTRODUCTION}

Curiosity and application have been driven scientists to explore stealth technology. It is known that an invisible plane, sometimes referred to as the stealth aircraft, is designed to reduce the reflection of electromagnetic waves radiated from radar, so that the plane cannot be detected or tracked [1-3]. The key problem for such research is how to minimize the aircraft's radar cross section. It is not easy to find much literature reporting on military camouflage due to confidentiality and safety considerations.

In addition to stealth aircraft technology, another kind of research on stealth technology concerns how to minimize the reflection of invisible light entering into a human's eye. It is well known that when visible light, reflected by some object, enters a human's eyes, the object is seen. Based on this fact, in order to make some object be invisible, the only thing needed is to bend the light escaping from our eyes. The common way to curl the light is through the use of refractive materials or reflection mirrors. Therefore, the core problem of stealth is how to bend the light efficiently in light of practical problems [4-10]. However, when using refractive material, it is inevitable that to some extent, there will be chromatic aberration image, color distortion and spherical aberration. All of these influence the stealth effect considerably. 
International Journal of Instrumentation and Control Systems (IJICS) Vol.7, No.3, July 2017

In this paper a novel approach, based only on the reflection of light and without using any other outer materials to camouflage a quadrangular prism is described. One essential and unique application is also introduced briefly.

\section{THE STRUCTURE OF A QUADRANGUlar PRISM OF SELF INVISIBILITY}

The reflective light bounced from any object goes into human's eye appears V-shaped as shown in Fig. 1 from which the shape of prism is selected to be the object camouflaged. A quadrangular prism of self-invisibility is defined as a quadrangular prism that can camouflage itself on its own, not by any other traditional rounding refractive materials or reflected mirrors or metamaterials as shown in Fig. 2. Such a quadrangular prism consists of four triangular prisms. The heights of all base triangles of triangular prisms are the same and two of each lateral face of mirrors are shown in Fig.2 (4) (5) (6) (7) reflecting invisible light effectively. Two triangular prisms (1) one up and one (2) down, are attached on the thin rectangular prism of transparence (3). Another identical part on the right side is placed face-to-face and symmetrically to the left one. All angles of the base triangle of triangular prisms depend strictly and completely on the following three factors: (i) the size of the pillar; (ii) the height of the base triangle of triangular prism; (iii) separation between the pillar and the observer. Beams parallel to the rectangular prism are, according to the law of light reflection, reflected by the relevant lateral face in sequence shown in Fig. 3 and go out of the system as if there are not any opaque triangular prisms at all. Therefore, the whole quadrangular is camouflaged by itself with ease.

\section{ONE OF THE DIRECT EXTRAPOLATIONS}

The design depicted above is a generalized case when the separation between pillar and observer is limited. A direct extrapolation can be obtained easily and elegantly. The intersection angle of two triangular prisms systems shown in Fig. 2 changes as the separation between the pillar and observer changes. When distance of separation goes to infinity, the quadrangular prism converts into a rectangular prism and all the base triangles of triangular prisms become identical isosceles triangles as shown in Fig. 4. The path of incident beams and the light reflected by all the mirror faces are shown in Fig. 5, similar to Fig.3, but much simpler. In such a case of extrapolation of limitation, the relative position between two isosceles triangles becomes simple, being expressed by a following concise formula:

$$
\mathrm{D}=\mathrm{h}(1+\tan 2 \theta \cot \theta)
$$

where $\mathrm{D}$ is the vertical interval between two transparent rectangular prisms; $\mathrm{h}$ is the height of base triangle as shown Fig.6; $\theta$ equals 90 degrees minus the incident angle.

\section{DISCUSSION}

In addition to images formed by a human eye, the only other perfect image is formed by a plane mirror, without any chromatic aberration and all sorts of distortions and aberrations. Using natural crystals, the design reported in [10] seems to produce a good invisibility effect, but the objects that can be camouflaged are only as big as an ant or a grain of sand. Consequently, it is not practical at all. Another counterpart of invisibility reported by [11], similar in part to the 
extrapolation from this invisible quadrangular prism, seems to camouflage macroscopic objects, but an outer reflection mirror, failing to be camouflaged, is inevitably needed.

At present, automobile structure contains some rigid pillars, supporting the windscreen, glass windows and top roof, occupy a big area that blocks the sight of drivers and consequently does not allow drivers to get a clear view of the surroundings during driving. Based on this reality, the invisible quadrangular reported here aims to make all the pillars invisible. Similar supporting pillars are necessary in planes, ships and even buildings. There is a need to increase clear sight lines. The disadvantage of such a prism is the inherent directionality. The scope of invisibility goes down when the observation point shifts off the most-clear direction or separation. Fortunately during driving, the position the driver maintains is nearly always the same. Based on the above discussion, one conclusion is that such an quadrangular prism of self-invisibility is very useful for improving the quality of all the vehicles.

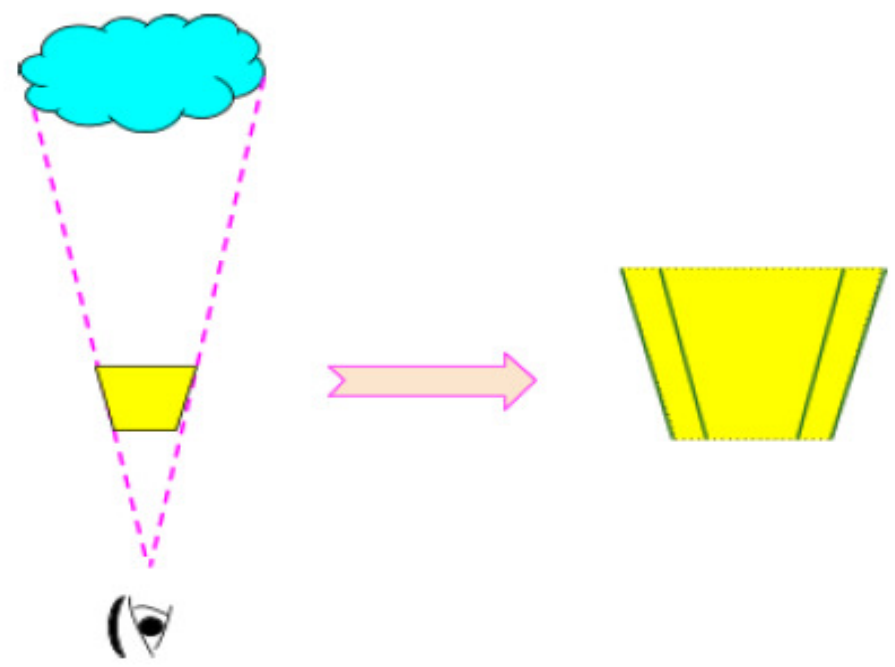

Fig.1 The line of sight towards objects seen appears V shape

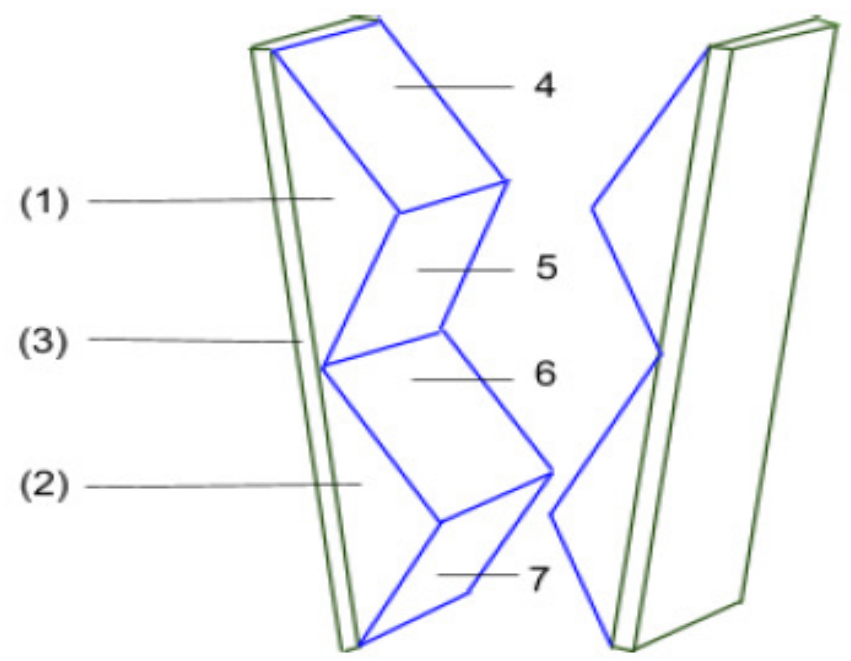

Fig. 2 Illustration of the invisible quadrangular prism 
International Journal of Instrumentation and Control Systems (IJICS) Vol.7, No.3, July 2017

(1) and (2) are triangular prisms with identical height of base triangle, but all the base angles should be adjusted well; (3) transparent rectangular prisms to fix (1) (2); (4) (4) (5) (6) are all mirrors to reflect visible light

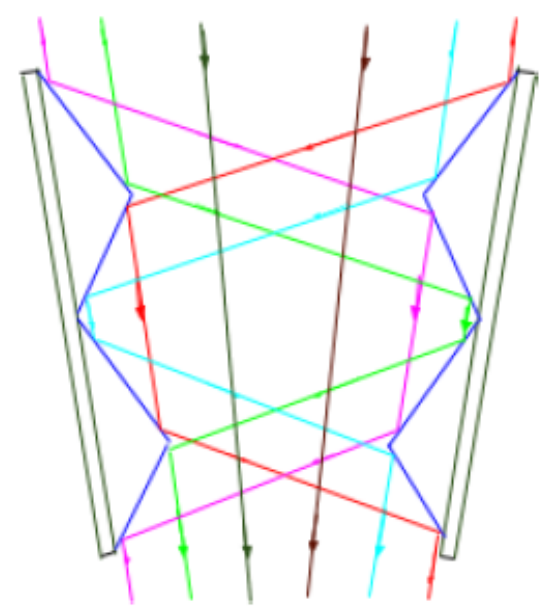

Fig. 3 Light path reflected in sequence by relevant mirror of lateral faces of all triangular prisms
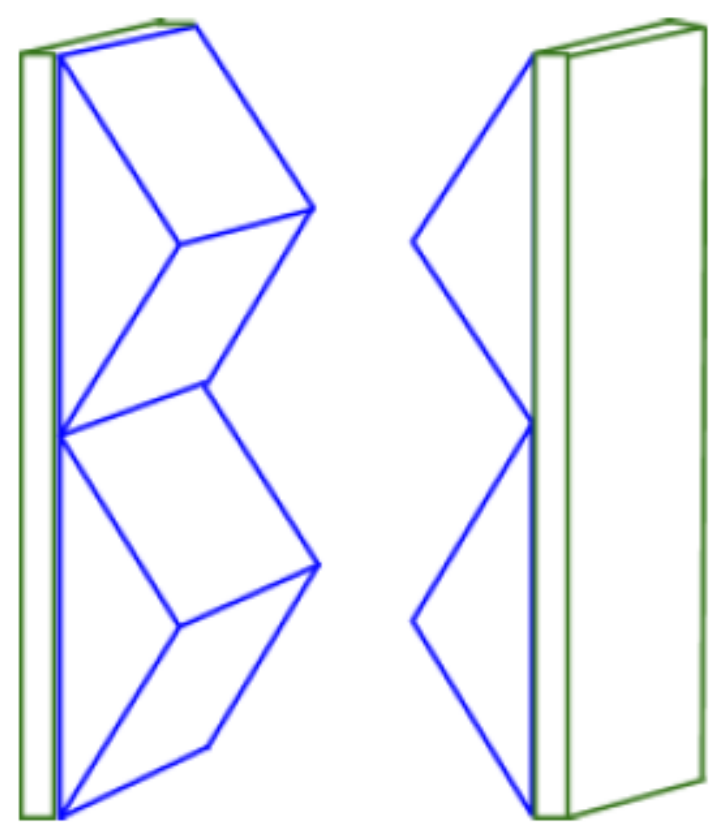

Fig. 4 When the distance between the pillar and the observer increases to the limitation of infinity, the quadrangular prism converts into a rectangular prism and all the base triangles of triangular prisms become isosceles triangles 
International Journal of Instrumentation and Control Systems (IJICS) Vol.7, No.3, July 2017

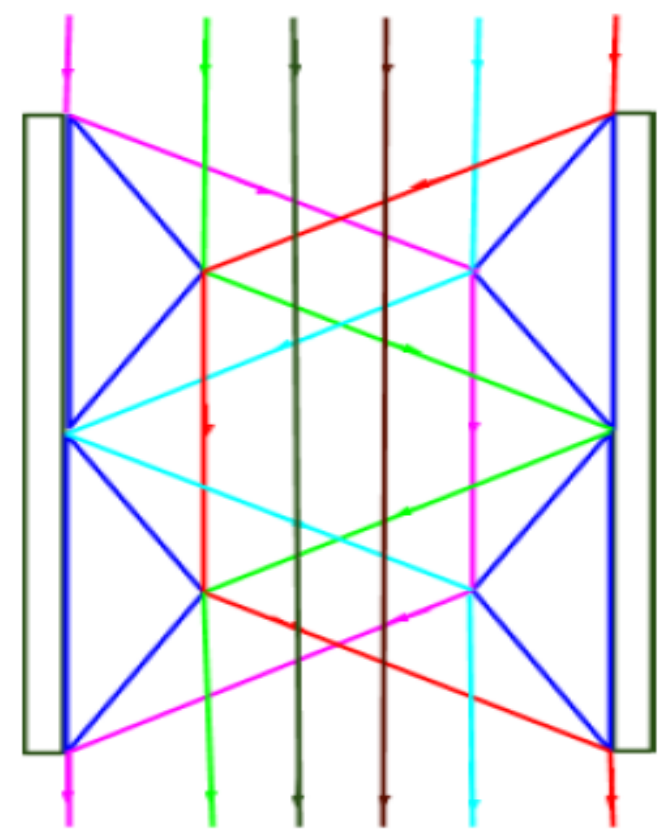

Fig. 5 Light path reflected in sequence by relevant mirrors of all the lateral face of rectangular prism

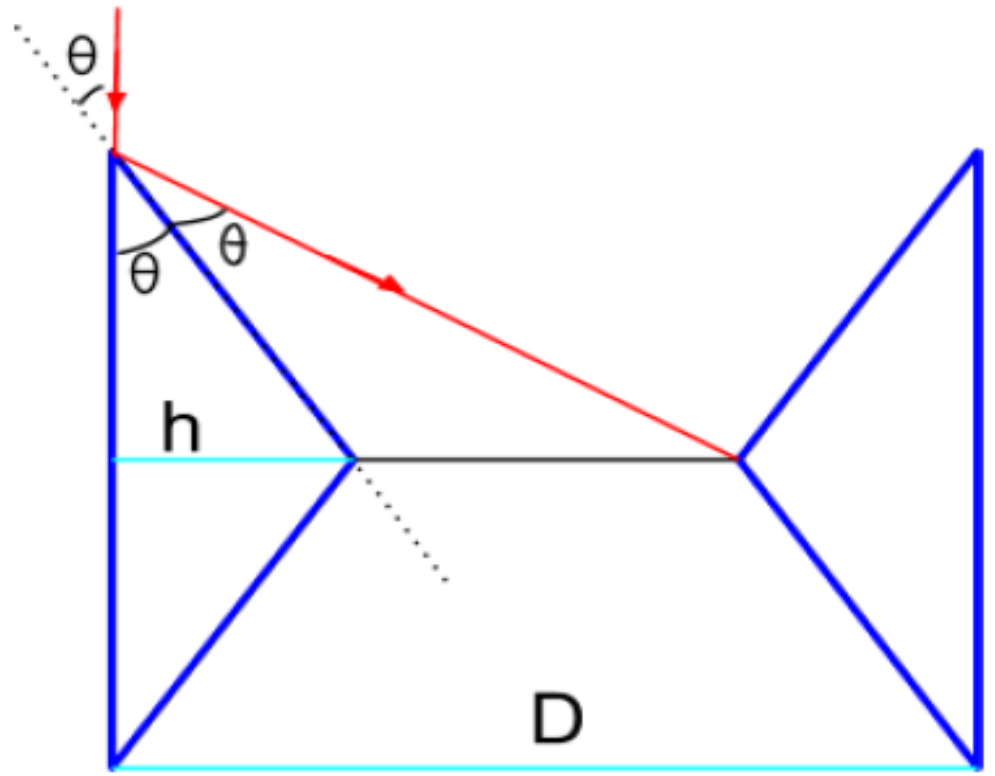

Fig. 6 Illustration the function relation between base angles of isosceles triangle and the separation of between two transparent rectangular prism $\mathrm{D}=\mathrm{h}(1+\tan 2 \theta \cot \theta)$ 
International Journal of Instrumentation and Control Systems (IJICS) Vol.7, No.3, July 2017

\section{REFERENCES}

[1] Mahulikar, S.P., Sonawane, H.R., \& Rao, G.A.: (2007) "Infrared signature studies of aerospace vehicles", Progress in Aerospace Sciences , v. 43 (7-8): 218-245.

[2] Rao, G.A., \& Mahulikar, S.P.: (2002) "Integrated review of stealth technology and its role in airpower", Aeronautical Journal , v. 106 (1066): 629-641.

[3] Myhra, David (July 2009). "Northrop Tests Hitler's 'Stealth' Fighter". Aviation History . 19 (6): 11.

[4] Wenshan Cai, Uday K, Chenttiar, etc, Optical cloaking with metamaterisal, nature photonics, vol.1, April 2007

[5] Schurig, D. et al, "Metamaterial electromagnetic cloak at microwave frequencies", Science 314, 977980 (2006).

[6] Christof Koch, Rendering the visible in visible, Scientific American mind, oct/nov. 2008

[7] Hongsheng Chen, etc, Ray-optics cloaking devices for large objects in incoherent natural light, NATURE COMMUNICATIONS | 4:2652

[8] Vivek Angoth, Amarjot Singh, M. Sai Shanka , A Novel Refractive Technique for Achieving Macroscopic Invisibility of Visual Light, Physics and Materials Chemistry, 2013, Vol. 1, No. 1, 5-8

[9] Pendry, J. B., Schurig, D. \& Smith, D. R. Controlling electromagnetic fields. Science $312,1780-1782$ (2006).

[10] https://www.wired.com/2011/02/invisibility-calcite-crystals/

[11] http://www.addictootech.com/invisibility-cloaking-device 